\title{
Analyses of a set of 128 ancestry informative single-nucleotide polymorphisms in a global set of 119 population samples
}

\author{
Judith R Kidd ${ }^{1 *}$, Françoise R Friedlaender ${ }^{2}$, William C Speed ${ }^{1}$, Andrew J Pakstis ${ }^{1}$, \\ Francisco M De La Vega ${ }^{3}$, Kenneth K Kidd ${ }^{1}$
}

\begin{abstract}
Background: Using DNA to determine an individual's ancestry from among human populations is generally interesting and useful for many purposes, including admixture mapping, controlling for population structure in disease or trait association studies and forensic ancestry inference. However, to estimate ancestry, including possible admixture within an individual, as well as heterogeneity within a group of individuals, allele frequencies are necessary for what are believed to be the contributing populations. For this purpose, panels of ancestry informative markers (AIMs) have been developed.
\end{abstract}

Results: We are presenting our work on one such panel, composed of 128 ancestry informative single-nucleotide polymorphisms (AISNPs) already proposed in the literature. Compared to previous studies of these AISNPs, we have studied three times the number of individuals $(4,871)$ in three times as many population samples $(119)$. We have validated this panel for many ancestry assignment and admixture studies, especially those that were the rationale for the original selection of the 128 SNPs: African Americans and Mexican Americans. At the same time, the limitations of the panel for distinguishing ancestry and quantifying admixture among Eurasian populations are noted.

Conclusion: We demonstrate the simultaneous importance of the specific set of population samples and their relative sample sizes in the use of the structure program to determine which groups cluster together and consequently influence the ability of a marker panel to infer ancestry. We demonstrate the strengths and weaknesses of this particular panel of AISNPs in a global context.

\section{Background}

In recent years, there have been many proposed ancestry informative markers (AIMs) and published sets of AIMs useful for particular purposes. Some sets have focused on estimating the admixture between specific ancestral populations such as the African and European genetic contributions to African Americans or European, Native American and African contributions to Latino populations [e.g., [1-7]. Others have focused on distinguishing ancestral origins from three or four continental regions, such as sub-Saharan Africa, Europe, East Asia and the Americas [8-12], or more broadly between many

\footnotetext{
* Correspondence: Judith.Kidd@yale.edu

'Department of Genetics, Yale University School of Medicine, 333 Cedar

Street, New Haven, CT 06510, USA

Full list of author information is available at the end of the article
}

globally distributed populations [13-16]. Yet others have focused on identifying the stratification of populations within particular geographic areas [e.g., [17-19] or within a clinical association study sample [20-22]. Whatever the purpose, the general usefulness of such AIMS depends very much on the set of populations used to identify and characterize them. Some global studies have used only a few but widely separated population samples [e.g., [15]. Others have used the HGDP-CEPH panel [23] of about 1,000 samples from 52 populations to select AIMs [10,11]. All approaches provide useful data but may also have weaknesses due to sampling error, either because the population samples used may not be highly representative of a broader geographic area or because the individual sample sizes are very small and subject to very large sampling errors. The same criticism applies to 
studies attempting to identify markers that provide ancestry information within a region, such as East Asia [24] or Europe [17,18].

Whatever the strategy for identifying them, AIMs are necessarily selected because they distinguish the specific population samples used. Therefore, replication with other samples of individuals from the same and/or closely related populations is necessary to verify the robustness of any set of AIMs. Such replication is onerous, costly and rarely undertaken.

Given the broad interest in AIMs in genetics, medicine, anthropology and forensics, the development of an optimal set of AIMs for a broad range of uses needs to be based on multiple markers studied on moderate to large samples of multiple relevant populations; appropriate resources will probably not be available in any single lab. As we advocated in the case of single-nucleotide polymorphisms (SNPs) for individual identification $[25,26]$, multiple labs need to test candidate markers on additional populations and for general robustness in the laboratory. While very large numbers of markers can provide quite accurate ancestry information for multiple geographic regions, small but robust sets of markers are especially useful.

Seldin's group [6,27] identified a set of 128 SNPs that they showed is useful for identification of the continental origin of people and in estimating the admixture proportions of these individuals. Thus, a particular aim was to develop a set of SNPs whose allele frequencies had major differences between the continental populations for use in matching controls and subjects in association studies. They validated all and various subsets of the 128 SNPs in their initial study of 825 individuals from 20 designated populations and subsequently in a study of 1,620 individuals from 48 population samples using a subset of 93 of the 128 SNPs [27].

Understanding that a set of AIMs (or ancestry identification SNPs, AISNPs) will only be broadly useful for population relationships and for identifying admixture if that set can be shown on a very large data set to be valid, we have tripled the size of the Nassir et al. [27] population set, increased the number of population samples to 119 and analyzed this sample with the 128 AIMs of Kosoy et al. [6]. We find that this set of 128 AISNPs is not only globally informative for origins from major geographic regions but also informative for distinguishing relationships within several of those regions. This provides further support for the usefulness of this set of SNPs in some ancestry/admixture analyses. We also note that these AISNPs are not particularly good at distinguishing within certain groups of populations, and a comparison of the Nassir et al. [27] results with ours illustrates effects of choice and size of the population samples analyzed.

\section{Methods}

\section{Samples}

We assembled a data set of samples of 4,871 individuals: those from the HapMap 3 [28], the Human Genome Diversity Project (HGDP) [29,30] and our lab, all typed for the 128 SNPs of Kosoy et al. [6]. Some of the HGDP samples used by Nassir et al. [27] are also included in our study, and for some of their populations we have an independent sample, e.g., Ashkenazi Jews. The HGDP contains 355 DNA samples from our lab or from cell lines we hold and routinely type in our lab and another 31 HGDP DNA samples are DNA samples we also have in our lab. When an HGDP sample is a subset of one of our population samples, we used only our inclusive sample. When a sample from our lab overlapped with an HGDP sample, the duplicates were removed from our sample and the full HGDP sample was included separately from our supplementary sample. Thus, we occasionally have two samples from the same population (e.g., Druze, PNG, Makrani), but no individuals from the two samples overlap. Sixteen populations are represented by two to four samples. Some of the "duplicate" populations (e.g., Han, Russians, Maasai) were sampled in different areas or countries, and some of the "duplicate" populations are independent samples from the same locale. Finally, the offspring in the HapMap 3 samples (ASW, CEU, MKK, YRI, and MEX) were removed so that the samples include only unrelated people. Table 1 provides the name, sources of the data and sample size for each of the final set of 119 population samples. All samples from our lab were collected with informed consent under protocols approved by the Institutional Review Board (IRB) at Yale University and other relevant IRBs; the other data are in the public domain. Descriptions of all of the population samples are in ALFRED [28] associated with the allele frequencies.

\section{Marker Data}

The polymorphic sites were those reported by Kosoy et al. [6]. The 3,071 samples from our lab were typed by TaqMan SNP Genotyping Assays ${ }^{\circledR}$ (Applied Biosystems, Foster City, California, USA). The HGDP marker data were downloaded from http://hagsc.org/hgdp/files. html[31]. The HapMap data were downloaded from http://hapmap.ncbi.nlm.nih.gov/index.html.yo [28]. Of the 128 SNPs typed for 119 population samples, only 16 instances (one AISNP for one population) of missing data existed in the public data. Eleven SNPs in seven HapMap 3 populations do not have genotype data available, and our estimates for those frequencies do not significantly affect the PCA results (Additional File 1). 
Table 1 Name, source of data, and sample size for the 119 population samples*

\begin{tabular}{|c|c|c|c|c|c|c|c|}
\hline Population & Abbreviation & $\mathrm{N}$ & Source & Komi Zyriane & $\mathrm{KMZ}$ & 47 & Yale \\
\hline$\overline{\text { Biaka }}$ & $\mathrm{BIA}$ & 67 & Yale $^{*}$ & Chuvash & $\mathrm{CHV}$ & 42 & Yale \\
\hline Mbuti & $\mathrm{MBU}$ & 39 & Yale* & Makrani 1 & MKR-2 & 26 & Yale \\
\hline Mandenka & MND & 24 & HGDP* & Makrani 2 & MKR-1 & 25 & HGDP \\
\hline Lisongo & LSG & 8 & Yale & Kalash & KLS & 25 & HGDP* \\
\hline Yoruba & YOR & 77 & Yale & Brahui & $\mathrm{BRH}$ & 25 & HGDP \\
\hline YorubaYRI & YRI & 113 & HарМар* & Balochi & $\mathrm{BCH}$ & 25 & $\mathrm{HGDP}^{*}$ \\
\hline Ibo & $\mathrm{IBO}$ & 48 & Yale & Sindhi & SDI & 25 & HGDP \\
\hline Zaramo & ZRM & 36 & Yale & Keralite & KER & 30 & Yale \\
\hline Hausa & HAS & 39 & Yale & Thoti & THT & 14 & Yale \\
\hline Bantu_NE & BTN & 12 & HGDP* & Kachari & $\mathrm{KCH}$ & 17 & Yale \\
\hline Bantu_S & BTS & 8 & $\mathrm{HGDP}^{*}$ & Gujarati GIH & $\mathrm{GlH}$ & 88 & НарМар \\
\hline San & SAN & 6 & HGDP* & Pathan 1 & PTH-1 & 75 & Yale \\
\hline Luhya LWK & LWK & 90 & НарМар & Pathan 2 & PTH-2 & 23 & HGDP \\
\hline African American 1 & AAM & 90 & Yale & Mohanna & MHN & 48 & HGDP \\
\hline African American ASW & ASW & 56 & HарМар & Burusho & $\mathrm{BSH}$ & 25 & $\mathrm{HGDP}^{*}$ \\
\hline Chagga & CGA & 45 & Yale & Khanty & KTY & 50 & Yale \\
\hline Maasai, T & MAS & 20 & Yale & Hazara 1 & HZR-1 & 87 & Yale \\
\hline Maasai MKK & MKK & 144 & HapMap & Hazara 2 & HZR-2 & 24 & HGDP \\
\hline Sandawe & SND & 40 & Yale & Uygur 2 & UYG & 10 & $\mathrm{HGDP}^{*}$ \\
\hline Ethiopian Jews & ETH & 32 & Yale & Uygur 1 & UIG & 45 & Yale \\
\hline Somali & SML & 12 & Yale & Khazak & KAZ & 44 & Yale \\
\hline Mozabite & $\mathrm{MOZ}$ & 30 & HGDP* & Khamba Tibetan & $\mathrm{KHG}$ & 27 & Yale \\
\hline Kuwaiti & KWT & 16 & Yale & Mongolians 1 & MVF & 62 & Yale \\
\hline Samaritans & SAM & 40 & Yale & Mongolians 2 & MGL & 10 & HGDP* \\
\hline Yemenite Jews & YMJ & 42 & Yale & HmongBlack & $\mathrm{HMQ}$ & 46 & Yale \\
\hline Palestinian 1 & PLA-1 & 49 & Yale & BaimaDee & $\mathrm{BQH}$ & 40 & Yale \\
\hline Palestinian 2 & PLA-2 & 51 & HGDP* & Qiang & QMR & 38 & Yale \\
\hline Druze 1 & DRU-1 & 75 & Yale & Hlai & LIC & 47 & Yale \\
\hline Druze 2 & DRU-2 & 47 & HGDP* & Yakut & YAK & 51 & Yale* \\
\hline Bedouin & BDN & 48 & HGDP* & Dai & DAI & 10 & HGDP \\
\hline Roman Jews & RMJ & 26 & Yale & Lahu & LHU & 10 & HGDP* \\
\hline Adygei & ADY & 54 & Yale $^{*}$ & Miaozu & MIZ & 10 & HGDP \\
\hline Greeks & GRK & 53 & Yale & Naxi & $N X I$ & 9 & HGDP \\
\hline Ashkenazi Jews & ASH & 79 & Yale & Oroqen & OQN & 10 & HGDP \\
\hline Tuscan 1 & Tus & 8 & HGDP & She & SHE & 10 & HGDP \\
\hline Tuscan TSI & TSI & 88 & Hapmap & $\mathrm{Tu}$ & TU & 10 & HGDP \\
\hline Sardinian 1 & SRD-1 & 34 & Yale & Tujia & TUJ & 10 & HGDP \\
\hline Sardinian 2 & SRD-2 & 28 & HGDP & Xibo & $\mathrm{XBO}$ & 9 & HGDP \\
\hline Orcadian & ORC & 16 & HGDP & Yizu & $\mathrm{YIZ}$ & 10 & HGDP \\
\hline North_Italian & ITN & 13 & HGDP & Daur & DUR & 9 & HGDP* \\
\hline French_Basque & FRB & 24 & HGDP* & Hezhen & HEZ & 9 & HGDP \\
\hline French & FRN & 29 & HGDP & Han, SF & HAN & 43 & HGDP \\
\hline Hungarians & $H G R$ & 89 & Yale & Han CHD & $\mathrm{CHD}$ & 85 & HapMap \\
\hline Irish & $|\mathrm{R}|$ & 114 & Yale & Han CHB & $\mathrm{CHB}$ & 84 & HapMap* \\
\hline European American 1 & EAM & 89 & Yale & Han, Taiwan & $\mathrm{CHT}$ & 50 & Yale \\
\hline European Amer CEU & CEU & 115 & HapMap* & Hakka & HKA & 41 & Yale \\
\hline Russians 1 & $\mathrm{RUA}$ & 33 & Yale & Koreans & KOR & 54 & Yale \\
\hline Russians 2 & RUV & 47 & Yale* $^{*}$ & Japanese & JPN & 50 & Yale \\
\hline Finns & $\mathrm{FIN}$ & 34 & Yale & Japanese JPT & JPT & 86 & HapMap* \\
\hline Danes & DAN & 51 & Yale & Laotians & LAO & 118 & Yale \\
\hline
\end{tabular}

Table 1 Name, source of data, and sample size for the 119 population samples* (Continued) 
Table 1 Name, source of data, and sample size for the 119 population samples* (Continued)

\begin{tabular}{|c|c|c|c|}
\hline Cambodians & CBD & 24 & Yale* \\
\hline Ami & AMI & 40 & Yale \\
\hline Atayal & ATL & 42 & Yale \\
\hline Malaysians & $M L Y$ & 11 & Yale \\
\hline Micronesians & MCR & 34 & Yale \\
\hline Samoans & $\mathrm{SMO}$ & 8 & Yale \\
\hline P-NG 1 & PNG & 13 & Yale \\
\hline P-NG 2 & PNG & 17 & $\mathrm{HGDP}^{*}$ \\
\hline Nasioi & NAS & 22 & Yale \\
\hline Mexican Amer MEX & MEX & 49 & HapMap* \\
\hline Pima Mexico & PMM & 53 & Yale* \\
\hline Maya & MAY & 51 & Yale $^{*}$ \\
\hline Quechua & QUE & 22 & Yale \\
\hline Colombians & $\mathrm{COL}-2$ & 13 & HGDP* \\
\hline Guihiba & $\mathrm{COL}-1$ & 11 & Yale \\
\hline Ticuna & $\mathrm{TIC}$ & 65 & Yale \\
\hline Surui R & SUR & 45 & Yale \\
\hline Karitiana & KAR & 55 & Yale \\
\hline
\end{tabular}

*These or subsets of these samples were included in Nassir et al. (2008). Descriptions of the populations and samples are in ALFRED.

\section{Fst}

Fst was calculated across all populations for each marker using the simple formula of Wright [32]: $\sigma^{2} / \overline{p q}$. For comparison, Fst was calculated for 2,327 other polymorphisms typed on our samples. None of these 2,327 polymorphisms included sites specifically selected for admixture or ancestry identification, or for individual identification; instead, they were all selected for other ongoing projects in our lab (i.e., linkage disequilibrium, disease/disorder association).

\section{PCA}

Principal component analysis (PCA) analyses of population sample allele frequencies were performed using XLSTAT (version 2009.4.07; Addinsoft SARL, http:// www.xlstat.com/en/company/)) as one method to evaluate effectiveness of these SNPs for distinguishing among populations and to determine the major factors accounting for the population frequencies.

\section{Structure}

Structure (version 2.3.3; software freely available at http:// pritch.bsd.uchicago.edu/structure.html[33-35]) was also used to evaluate and illustrate the effectiveness of these sites to distinguish among these populations. The burnin was set at 20,000 followed by 10,000 iterations, and a model of correlated allele frequencies was specified. Ten replicates at each "K" levels 2-6 and 20 replicates at $K=7$ and $K=8$ were evaluated using CLUMPP; (software freely available at http://rosenberglab.bioinformatics.med. umich.edu/clumpp.html) [36]. Specific solutions have been plotted using DISTRUCT 1.1; software freely available at http://rosenberglab.bioinformatics.med.umich. edu/distruct.html) [37]. The matrix of pairwise similarities among replicate runs was used to identify different overall patterns based on high $\mathrm{G}$ values among runs with the "same" pattern and lower values for runs with different patterns.

\section{Results \\ New data}

The allele frequencies for the 128 AISNPs for all 119 population samples are given in Additional file 2, and the allele frequencies of the 69 population samples tested in our lab have all been entered into the ALFRED database [30] and can be readily accessed using the rs\# of each SNP.

\section{Fst}

The Fst distribution of the 128 AISNPs was compared to the distribution of 2,327 non-AISNPs typed in our lab (Figure 1 and Additional file 3). Although Kosoy et al. [6] selected their 128 AISNPs not on the basis of Fst, but rather on the Informativeness statistic $\left(I_{n}\right)$ of Rosenberg et al. [38,39], Fst clearly separates the two distributions by 1.25 standard deviations. The null hypothesis that the two distributions are the same is rejected with a probability considerably less than 0.001 . Outliers in the two distributions are given in Additional file 4. At the high-Fst end of the distributions, there are nine sites with Fst greater than 0.48: seven are in the reference distribution, and two are in the AISNP distribution. Of the seven in the reference distribution, five are located in or near genes of known phenotypic effect (SLC24A5, OCA2 (two SNPs), HERC2 and EDAR), and each of these genes is well known to have SNPs with marked global variation in allele frequency; but the best "known" SNPs are not part of this 128 AISNP set (Additional file 4). Though not associated with a phenotype, the remaining two "outliers" in the reference distribution have comparably high Fst values (Additional file 4). The two outliers at the high end of the AISNP distribution are sites in or near EDAR (rs260690, Fst $=0.5205)$ and RTTN (rs4891825, Fst $=0.5176)$. There are 10 outliers at the low end of the reference Fst distribution with Fst $<0.04$. Only one of the AISNPs falls below the mode of the reference distribution: TWGS1 (rs4798812, Fst $=0.08753)$.

\section{PCA}

Figure 2 presents the first three factors of the PCA analysis based on allele frequencies of each of the 119 samples. The first two factors account for more than $72 \%$ of the variance. Factor 3 accounts for an additional $8.7 \%$ of 


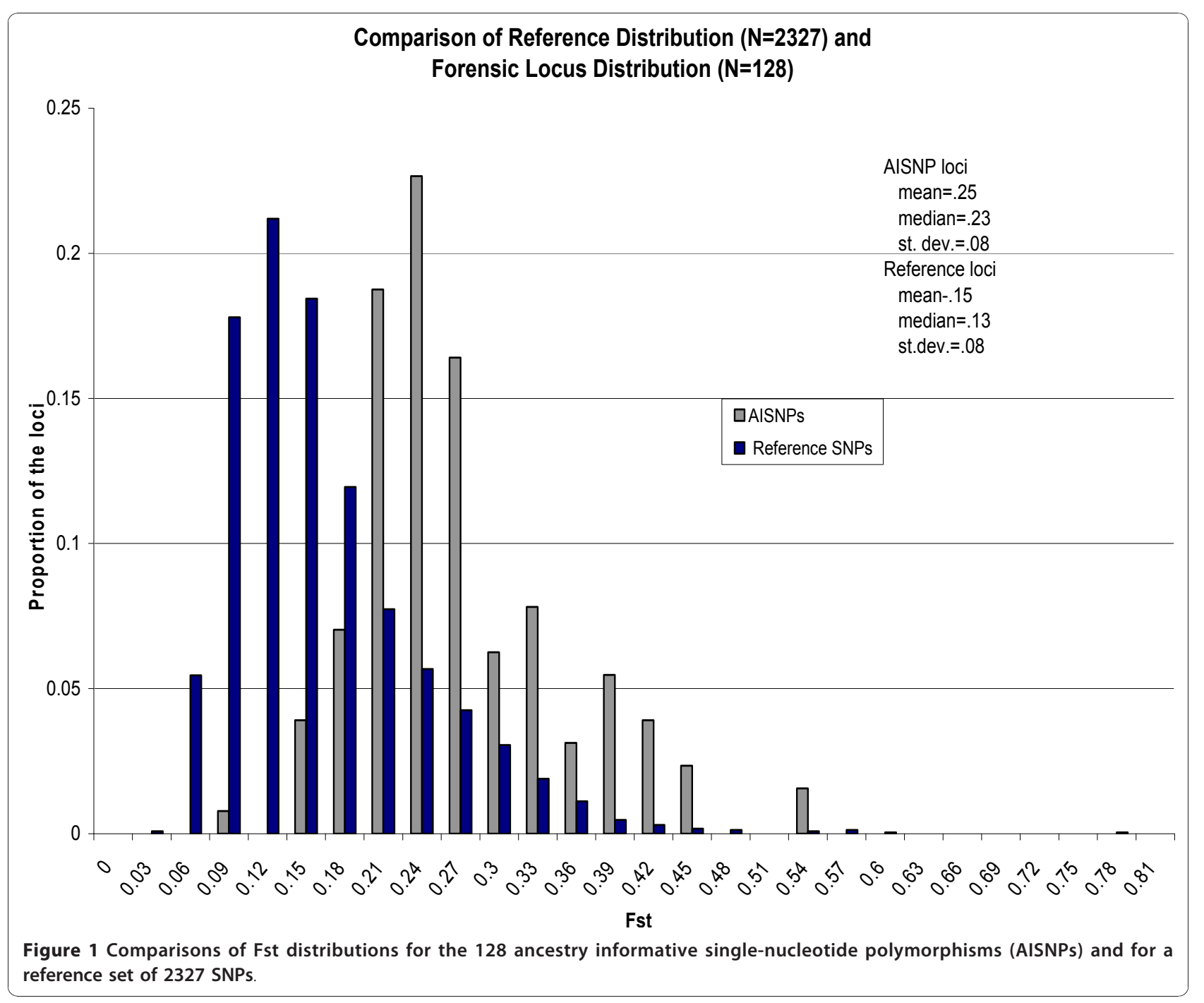

the total variance. Factor 1 clearly separates the Native Americans from all other groups, and factor 2 clearly separates the African populations from the rest. Factor 3 emphasizes the difference between Native Americans and East Asians. This set of AISNPs was selected by Kosoy et al. [6] to maximize the differences among European Americans, Africans and Native Americans; those three groups clearly are at the vertices of the triangular pattern based on factors 1 and 2 (Figure 2). We also note that Eurasian populations show less clear separation.

\section{Structure}

Results for the specific structure runs with the highest likelihood at each $K$ value, $K=2-8$, are shown in Figure 3 along with the number of times the particular overall pattern occurred. At all the $K$ values, there are populations in each of the groups that seem quite homogeneous. By $K=8$, the likelihoods began to plateau (Additional file 5), providing a statistically reasonable stopping point. For a better understanding of the ability of the data to distinguish most likely ancestry at the higher $K$ values, we ran structure a total of 20 times, and the patterns seen more than once are illustrated in Figure 4; the patterns and likelihoods of the individual runs are given in Table 2. As is obvious from the patterns and the likelihoods of the individual runs, some distinctions are quite consistent while others generate similar likelihoods with combinatorial alternatives for a few different groups of populations.

At $K=7$, there is no single solution clearly identifiable as best. Five different overall patterns occur in the 20 runs. The pattern illustrated in Figure 3 has the highest likelihood but is not the most common pattern. The next highest likelihood is nearly identical and occurs for a pattern that occurs in 6 of the 20 runs and differs by subdividing East Asian populations and not distinguishing the Pacific populations. The most frequent pattern, found for 

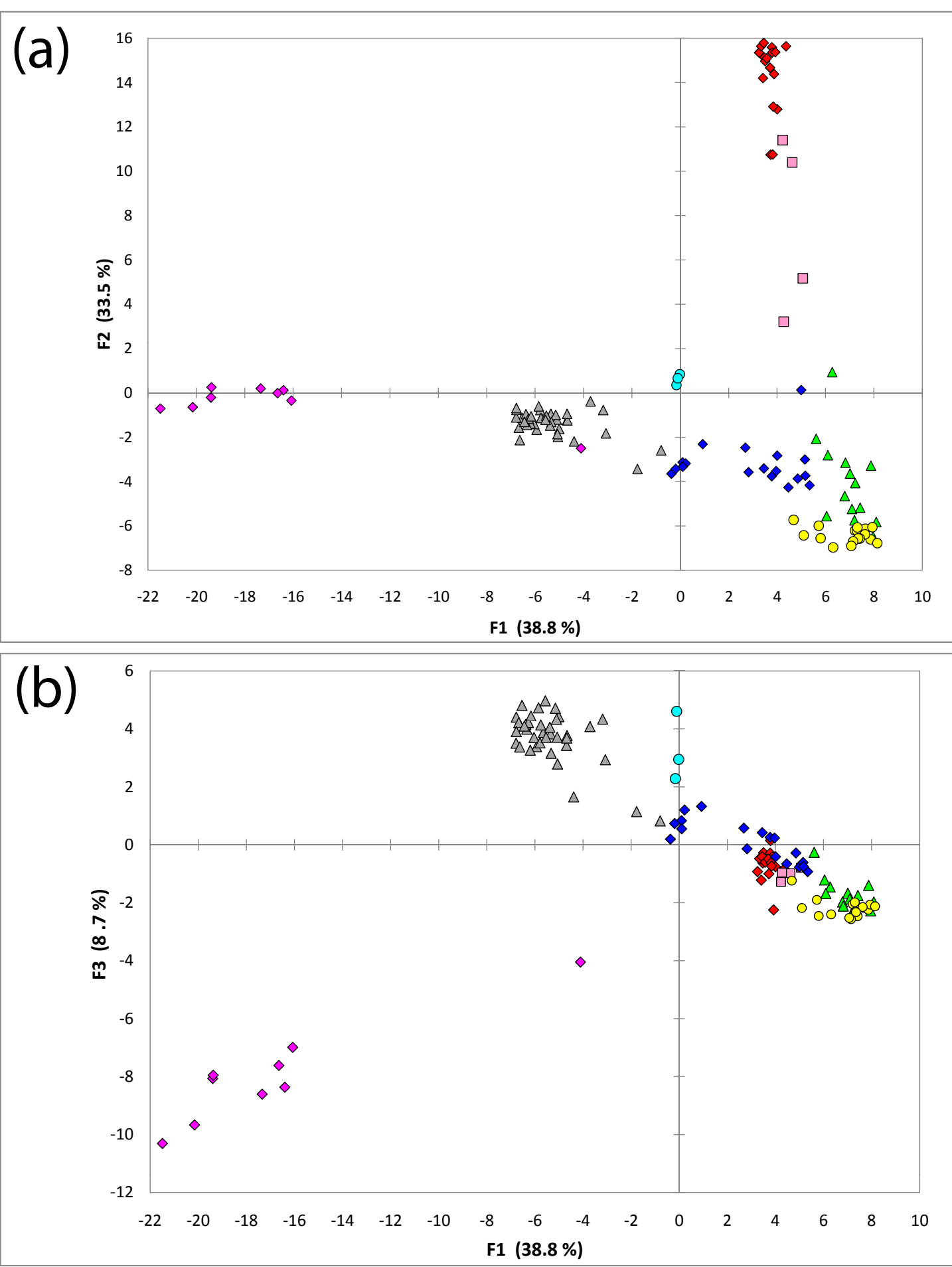

Figure 2 Principal component analysis (PCA) of 119 population samples based on allele frequencies of 128 AISNPs.

7 of the 20 runs, does not have the highest likelihoods and differs in separating East African populations from the Pacific populations.

At $K=8$, eight different overall patterns occur among the 20 separate runs. The pattern shown is the most commonly found and does have the highest likelihood among the 20 runs. A nearly equal likelihood occurred for a somewhat different overall pattern that subdivides East Asia rather than separating East African populations. 


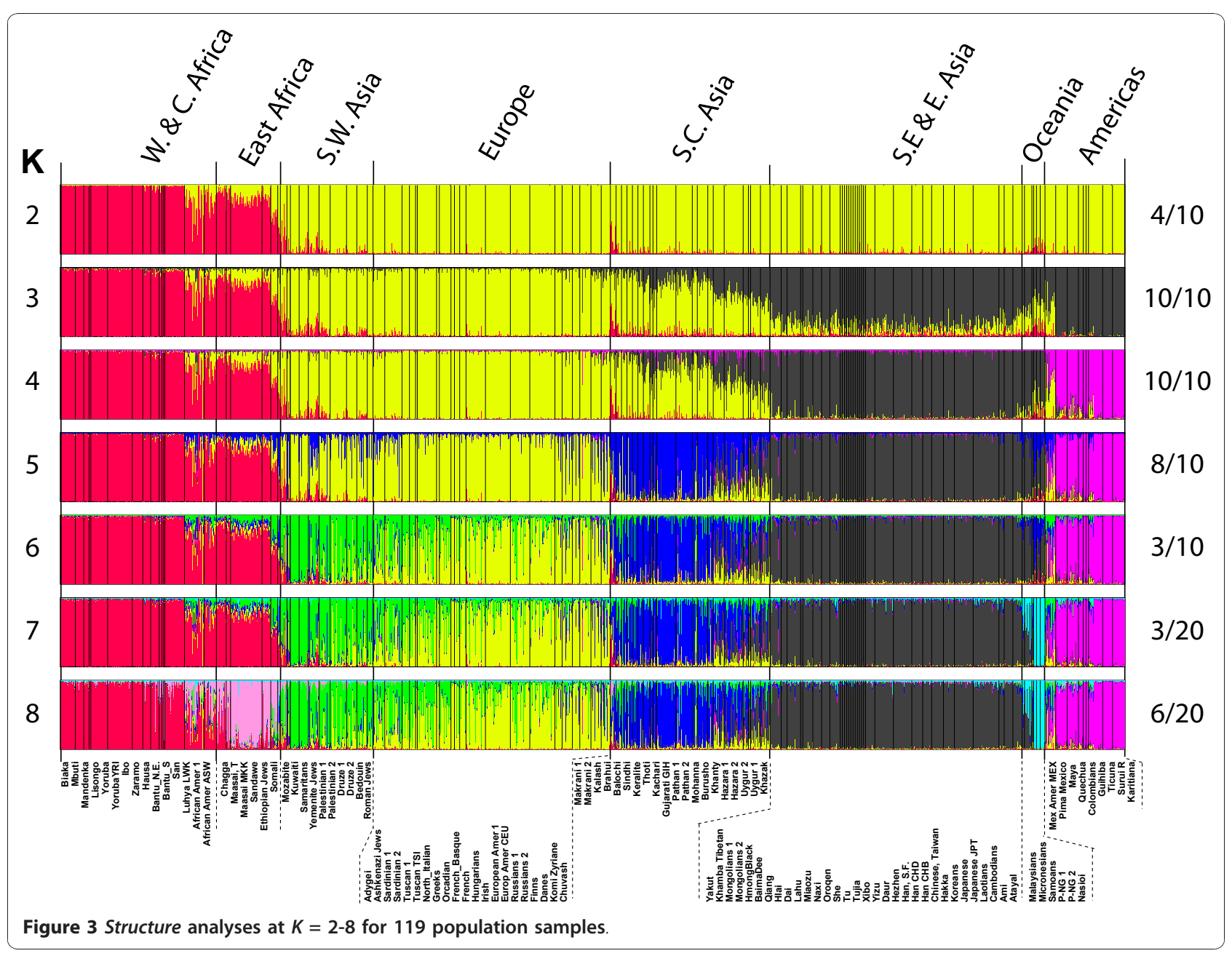

At $K=8$, the results can be summarized with respect to the pattern shown in Figure 3 (pattern A in Figure 4). Starting from the left, the sub-Saharan Africans, especially the West Africans, seem relatively homogeneous (red). The East Africans, especially the MKK, Sandawe and Ethiopian Jews, can form a distinct grouping (pink), in which case other Tanzanian populations, the Maasai and Chagga, and the Somali (now living in Pakistan) appear intermediate between East and West Africa. The next consistent cluster includes the Mozabites and Southwest Asians (green). There is then a more-or-less gradient across Europe from southeastern and southern Europe (mostly green), through to northwestern Europe, and ending in northeastern Europe (mostly yellow). The south-central Asian populations form another (dark blue) consistent and relatively homogeneous cluster of populations, including East Indians and several Pakistani populations (dark blue). The Khazaks, Uyghur, Hazara and Khanty form a "group" that is depicted as admixed under any of the alternative common patterns. The next group of populations (dark gray) appears homogeneous from the Khamba-Tibet through Southeast Asia all the way to East Asia but the alternative (pattern B in Figure 4) has the western Chinese groups at one end of a more clinal pattern with the southeastern Asians at the other end. Interestingly, this alternative depicts the Han, Koreans and Japanese as admixed. The next clear cluster (light blue) is Pacific and consists of three Melanesian samples: The Samoans, Micronesians and Malaysians appear intermediate between East Asia and the Pacific. The final clear cluster (pink) consists of Native American samples.

The different patterns at $K=7$ and $K=8$ show fine distinctions even among the regions that are superficially similar. To make some of these clearer, we have generated the population averages for the best result (highest likelihood) for each of the patterns (Figure 5). These emphasize the variation among individuals in each population sample by showing the population as multiple colors. These figures also emphasize the southwestern Asia through northern Europe cline seen in all patterns.

Clusters that emerge at even higher values of $K$ include a Pygmy/San/S Bantu cluster in Africa, a Khanty/ 


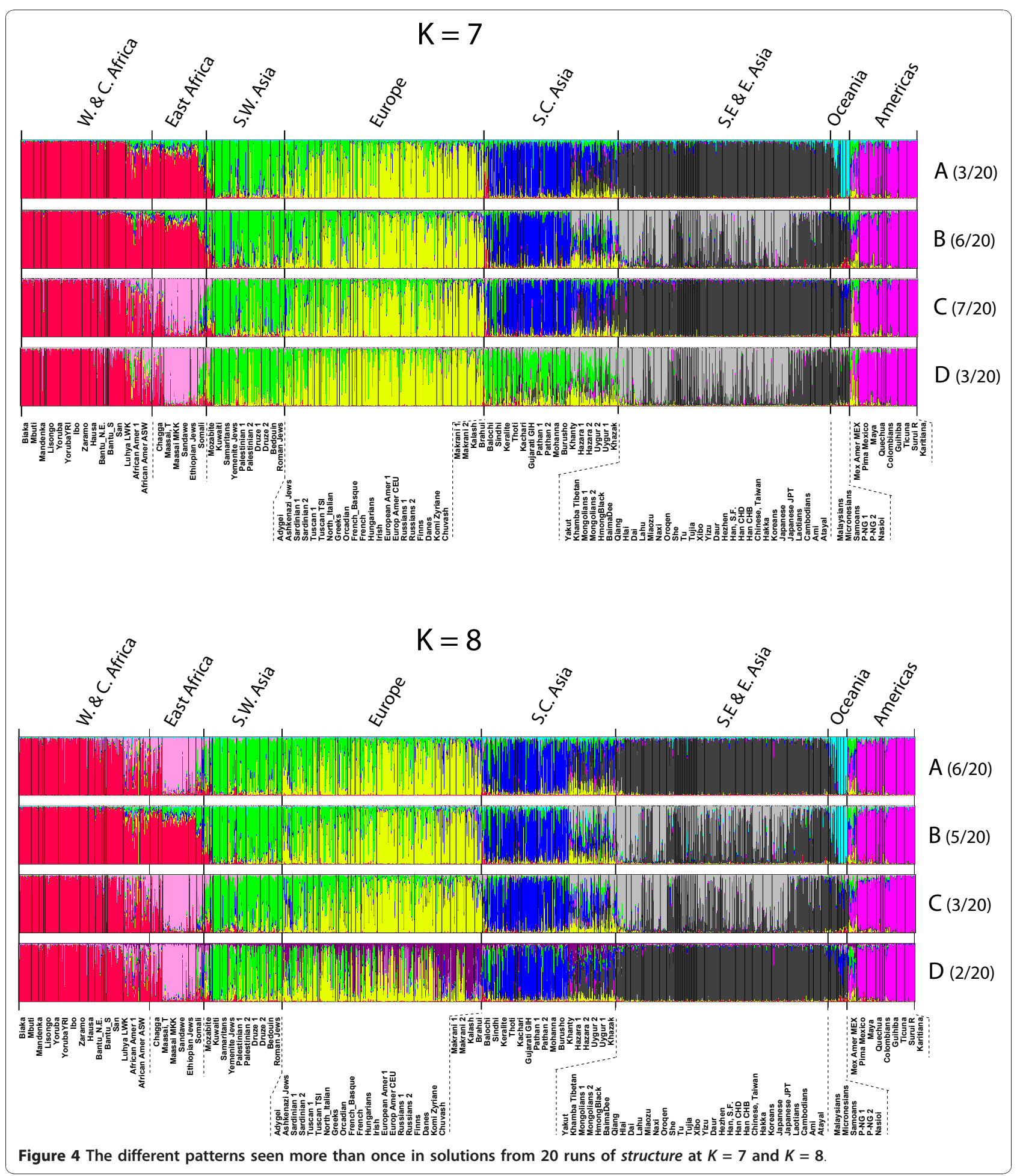

Khazak/Yakut cluster in Asia and a vaguely central Asian group consisting of, for example, the KhambaTibet, Mongolian, Baima Dee and Qiang. These clusters, though reasonable, are not strongly supported statistically.

\section{Discussion}

A set of markers particularly useful for determining in detail the genetic distinctions among populations should also be useful in an examination of admixture. However, "admixture" is not a singular phenomenon: A sample of 
Table 2 Patterns and likelihoods of 20 structure runs at $K=7$ and $K=8$

\begin{tabular}{cllcclll}
\hline Pattern $K=\mathbf{7}$ & LnP(D) & Run & Best per pattern & Pattern $\boldsymbol{K}=\mathbf{8}$ & LnP(D) & Run & Best per pattern \\
\hline A & -591354 & run13 & $*$ & A & -590090 & run13 \\
B & -591528 & run1 & $*$ & B & -590185 & run1 \\
B & -591555 & run2 & & B & -590570 & run6 \\
A & -591571 & run3 & & B & -590605 & run16 \\
B & -591707 & run12 & & A & -590606 & run15 \\
B & -591724 & run8 & & C & -590867 & run4 \\
C & -591822 & run7 & $*$ & A & -591033 & run18 \\
A & -591855 & run17 & & A & -591053 & run2 \\
B & -591944 & run15 & & C & -591080 & run20 \\
C & -591949 & run5 & & A & -591090 & run10 \\
C & -591957 & run9 & & E & -591160 & run5 \\
C & -592012 & run11 & & C & -591298 & run14 \\
B & -592017 & run4 & & C & -591371 & run3 \\
D & -592137 & run20 & $*$ & D & -591512 & run7 \\
D & -592272 & run18 & & D & -591689 & run17 \\
E & -592309 & run6 & & A & -591744 & run12 \\
C & -592342 & run16 & & C & -591745 & run8 \\
C & -592548 & run19 & & F & -592008 & run19 \\
C & -592605 & run14 & G & -592162 & run11 \\
D & -593102 & run10 & & H & -592261 & run9 \\
\hline
\end{tabular}

individuals might be considered admixed if it is composed of (1) samples from two or more different populations, (2) the descendants of people from two or more populations who have "recently" intermarried, (3) descendants of people from two or more populations who have intermarried in the ancient past and (4) people discretely sampled from a single region along a geographic allele frequency cline established predominantly by random genetic drift.

The American Society of Human Genetics Ancestry and Ancestry Testing Task Force, in its white paper [40], sets forth caveats to be kept in mind in ancestry inference, perhaps the foremost of which is that ancestral populations cannot be observed directly and that even surrogates for those ancestral populations may not be included in any given study. Therefore, the "gold standard" analytic programs such as structure (version 2.3.3; http://pritch.bsd.uchicago.edu/structure.html [32-34]) will cause individuals in some populations to appear as an "admixture" of the population samples that are in the analysis. Even in analyses of principal components, it is not possible to distinguish whether a population is admixed or simply intermediate. Thus, a set of AIMs estimating the ancestry of an individual whose ancestry involves populations other than the majority of the populations in an analysis may be unsatisfactory by forcing that individual to be explained by the ancestry inferred for the majority. Further, a set of AIMs selected for one set of populations cannot be expected to be as good at distinguishing among other populations, perhaps even from the same geographic regions.

It is important to realize that the outcome of any analysis of admixture or other population structure depends heavily on both the population samples and the markers used. Though we have included some of the same HGDP populations as Kosoy et al. [6] did in their analyses, the outcome is always a function of which samples are included. Thus, in our selection of samples, we have also included samples that overlap with those reported by Nassir et al. [27] as well as others not part of either the Kosoy et al. [6] or Nassir et al. [27] reports.

\section{PCA}

The results shown in Figure 2 clearly reflect the criteria used to select this set of AISNPs [6]. The strongest discrimination reflects the geographic and ancestral origins of those populations (Africa, Europe, East Asia and the Americas), even though this analysis included none of the original samples used to select the SNPs. The first two components provide strong support for these SNPs in studies involving African, European and Native American populations. The relatively poorer separation among Eurasian and Pacific populations reflects the absence of Central, South and East Asian and Pacific populations in the selection of these AIMs as well as their distinct evolutionary relationships relative to African and Native American populations. It is logical to expect that if more SNPs with large allele frequency 


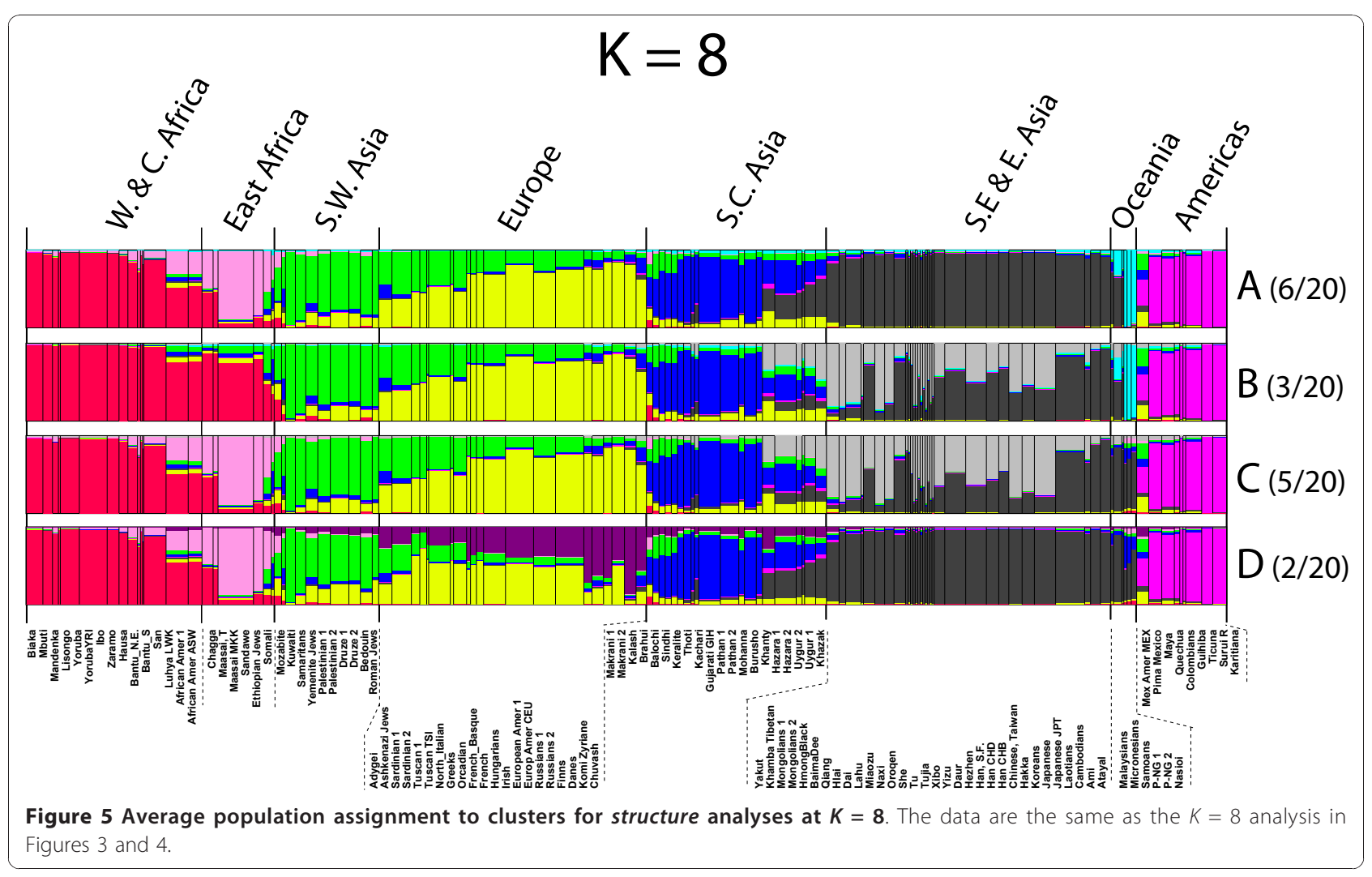

differences across Eurasia were included, factor 3 would show greater separation between west and east Eurasia.

\section{Structure}

Structure attempts to find the set of K population allele frequencies that will give the best fit to all individual samples assuming Hardy-Weinberg ratios for each of the K populations. Structure does not consider or produce analyses of population relationships. Fortunately, this is not an issue of interest to forensic science. Rather, structure assigns individuals to clusters of genetically similar individuals. Obviously, if numbers of individuals differ greatly among different populations, a population sample with a large number of individuals will influence the allele frequencies of the particular cluster into which it falls more than a population with a small number of individuals. Thus, a small population from the middle of a cline with larger numbers in populations from the more extreme parts of the cline will appear "admixed." Such is seen at $K=3$ for the South, Central and East Asian populations. However, a large population from that middle region will, at the same $K$ value, cause the allele frequency estimates of the flanking clusters to move toward the center even if cluster assignments do not change. The consequences at higher $\mathrm{K}$ values may be that the "middle" population is a distinct group or, by shifting the estimates for the flanking clusters, cause a population at the extreme of the cline to "fall off" and become a separate cluster. The cluster assignments at $K=4$ and $K=5$ illustrate this (Figure 3). In other words, conclusions about groupings at a given value of $K$ are a function of the populations sampled and their relative sample sizes. Thus, it is not necessarily correct that the estimated allele frequencies for a given cluster represent the ancestral population, nor can one automatically interpret a partial assignment to two or more clusters as admixture. In addition, as shown in Figure 4 and Table 2, there is a stochastic element in each structure run such that the relative likelihoods of different patterns from different runs depend on the particular outcomes that happen to occur. Thus, the point of using structure is not the single best run or the most common pattern seen, but the stability of aspects of the patterns and of the individual runs within each pattern among the runs with the higher likelihoods. Kalinowski [41] has recently published studies making additional relevant points on the interpretation of structure results.

An example of the sample size effect appears to be found in Nassir et al. [27]. That study contains 49 populations with a total sample size of 1,620. Their Ashkenazi sample of 240 individuals (two population samples pooled: Ashkenazi AM 4 GP and Ashkenazi AM) constitutes about $15 \%$ of the total. Similarly, the 259 European Americans (two population samples pooled: 
European AM CEU and European AM NYCP) constitute about $16 \%$ of the total. These two heavily weighted population samples probably decrease the resolution of European and southwestern Asian populations. Our data set with the same sites and no population consisting of more than $6 \%$ (Han, pooling four population samples, $\mathrm{CHB}, \mathrm{CHD}, \mathrm{SF}$ and Taiwan $=5.4 \%$ ) of the total sample can begin to distinguish a southwestern Asian cluster at $K=6$, though showing a cline through Europe. Unfortunately, almost all of our East Asian samples, including many Chinese minorities, are de facto similar, with this set of AISNPs constituting the equivalent of nearly a quarter of our whole sample through $K=8$, clearly affecting how South Asian and especially Central Asian populations appear. There are, however, differences among them sufficient to result in a more complex clinal pattern as a reasonable alternative at $K=7$ and $K=8$ (Figure 4). In the ideal world, a world we doubt exists, all samples would be large, equal in size and evenly distributed around the world.

\section{Forensic Implications}

Our analyses have been directed toward evaluating this set of SNPs for a particular purpose: ancestry inference as an investigatory tool. We have used PCA and structure for these evaluations. However, we do not advocate using either PCA or structure as a forensic tool for inference of individual ancestry in casework. Direct evaluation by likelihood methods is much more accurate. Any polymorphism can also be used to assist in matching crime scene and suspect DNA genotypes and to estimate the probability of the match occurring by chance if allele frequency data exist. Therefore, these 128 AISNPs could be used for exclusion, but we would not advise use of these markers to estimate the probability of a match occurring by chance. They have been selected to distinguish among populations and to have highly varying frequencies. To use these data in a court, one would have to present a diverse set of calculations and assumptions. The complexities of the calculations and the assumptions would allow an easy challenge, and all potential benefits of SNPs over the standard CODIS markers would be lost. There are good panels of SNPs selected for individual identification [e.g., [25,26]. The set of SNPs for individual identification that we developed [26] largely circumvents the problem of different allele frequencies in populations from different parts of the world. Similarly, we feel the 128 AISNPs analyzed in this paper are not efficient for any estimates of phenotype beyond the very indirect inference from ancestry.

The data for these SNPs can be used to "assign" regional ancestry to a single individual based on the genotypes at all or a significant fraction of these 128 SNPs.
This would be done by calculating the likelihood of the multisite genotype based on the allele frequencies of each of the 119 population samples (frequencies are in ALFRED [37]). It is clear that for many genotypes, many populations will have roughly comparable likelihoods. The clusters at $K=9-11$ (not shown) indicate no new strongly supported subgroups of populations and suggest, for example, that differentiating ancestry from among populations within East Asia will not be easy using the allele frequencies for this set of SNPs.

It is important to distinguish population averages from the variation among individuals (Additional files 6 and 7) within that population. Figure 5 presents the population averages for the $K=8$ structure analysis. Compared to the variation among individuals shown in Figure 4, the averages make some of the global patterns clearer but completely obscure the individual variation that can be of great importance in a forensic setting.

In a comparative examination of a total of seven small publicized AISNP panels containing a total of 688 SNPs, we found that only one SNP (rs2065160) occurred in three of the panels and 26 other SNPs (about 4\%) occurred in two panels. None of the 128 SNPs in the panel we have analyzed occurred in any of these other panels. The small number of overlapping SNPs across panels likely results from the different methods of selecting SNPs, the different data sets from which SNPs are selected and the different purposes of the panels: some are global, some are regional and some are for the four continental extremes. However, though the specifics of these panels are not relevant, it is clear that there is no single set of AISNPs that will be of value for all questions.

With our additional data and the analyses presented here, this panel of 128 AISNPs is the best documented and validated for broad global application to infer ancestry. However, it is not necessarily the optimal panel depending on the question being asked, and it is definitely not optimal at identifying ancestry within Europe and Southwest Asia (cf. Figures 3, 4 and 5; $K=6-8$ ). Distinguishing among East Asian populations is also not optimal with this set of AISNPs. Neither of these conclusions is surprising, since populations in those regions were not part of the selection of this set of AISNPs. Selection to identify SNPs with markedly different allele frequencies across East Asia will be necessary [24]. Many useful SNPs must already exist; the problem is to identify them. In general, as more and more SNPs are identified through ongoing sequencing projects, other SNPs may be optimal for resolving population similarities within one of the major clusters in the structure analyses of Figures 3 and 4. However, comparison of the relative discriminating ability of additional candidate SNPs requires that all SNPs be typed on the same 
populations and, ideally, the same individuals. That will require coordination among laboratories and sharing of data and/or samples. We have put all of the allele frequencies of the populations we have studied in this paper into ALFRED [30]; the raw individual genotype data are available on request.

\section{Additional material}

\section{Additional file 1: List of missing values and how they were handled in the PCA.}

Additional file 2: Population allele frequencies.

Additional file 3: List of Fst values for all 128 AISNPs.

Additional file 4: List of upper and lower outliers for Fst in

Reference and AISNP distributions.

Additional file 5: Likelihood plot $\mathrm{K}=2-12$

Additional file 6: Individual Assignments in Structure analysis

Additional file 7: Population Assignments in Structure analysis

\section{Acknowledgements}

This work was funded in part by National Institute of Justice grant 12007 DN-BX-K197 to Kenneth K Kidd. The points of view expressed in this document are those of the authors and do not necessarily represent the official position or policies of the U.S. Department of Justice. Part of this work (structure analyses) was carried out using the resources of the Computational Biology Service Unit from Cornell University, which is partially funded by Microsoft Corporation. We thank all the colleagues who helped us assemble the population samples, the Coriell Institute for Medical Research (National Institute of General Medical Sciences Human Genetic Cell Repository) and The National Laboratory for the Genetics of Israeli Populations at Tel-Aviv University. Special thanks are due to the thousands of individuals from these populations who volunteered to give blood samples for studies such as this one. We thank the reviewers for comments that helped improve this paper.

\section{Author details}

${ }^{1}$ Department of Genetics, Yale University School of Medicine, 333 Cedar Street, New Haven, CT 06510, USA. ${ }^{2}$ Independent researcher, Sharon, CT 06069, USA. ${ }^{3}$ Life Technologies, Foster City, CA 94404, USA.

\section{Authors' contributions}

All authors have read and approved the final manuscript. KKK and FMDLV were involved in the conception and design of the study, and KKK assisted in writing the manuscript. JRK supervised the genotyping assays and data analysis and wrote the manuscript. FRF and AJP assisted in the data analysis. WCS assembled and integrated the data set from the literature and laboratory.

\section{Competing interests}

The authors declare that they have no competing interests. FMDLV is employed by Life Technologies.

Received: 13 August 2010 Accepted: 5 January 2011

Published: 5 January 2011

\section{References}

1. Bryc K, Auton A, Nelson MR, Oksenberg JR, Hauser SL, Williams S, Froment A, Bodo JM, Wambebe C, Tishkoff SA, Bustamante CD: Genomewide patterns of population structure and admixture in West Africans and African Americans. Proc Natl Acad Sci USA 2010, 107:786-791.

2. Price AL, Patterson N, Yu F, Cox DR, Waliszewska A, McDonald GJ, Tandon A, Schirmer C, Neubauer J, Bedoya G, Duque C, Villegas A, Bortolini MC, Salzano FM, Gallo C, Mazzotti G, Tello-Ruiz M, Riba L,
Aguilar-Salinas CA, Canizales-Quinteros S, Menjivar M, Klitz W, Henderson B, Haiman CA, Winkler C, Tusie-Luna T, Ruiz-Linares A, Reich D: A genomewide admixture map for Latino populations. Am J Hum Genet 2007, 80:1024-1036.

3. Santos NP, Ribeiro-Rodrigues EM, Ribeiro-Dos-Santos AK, Pereira R, Gusmão L, Amorim A, Guerreiro JF, Zago MA, Matte C, Hutz MH, Santos SE: Assessing individual interethnic admixture and population substructure using a 48-insertion-deletion (INSEL) ancestry-informative marker (AIM) panel. Hum Mutat 2010, 31:184-190.

4. Lins TC, Vieira RG, Abreu BS, Grattapaglia D, Pereira RW: Genetic composition of Brazilian population samples based on a set of twentyeight ancestry informative SNPs. Am J Hum Biol 2010, 22:187-192.

5. Collins-Schramm HE, Chima B, Operario DJ, Criswell LA, Seldin MF: Markers informative for ancestry demonstrate consistent megabase-length linkage disequilibrium in the African American population. Hum Genet 2003, 113:211-219.

6. Kosoy R, Nassir R, Tian C, White PA, Butler LLM, Silva G, Kittles R, AlarconRiquelme ME, Gregersen PK, Belmont JW, De La Vega FM, Seldin MF: Ancestry informative marker sets for determining continental origin and admixture proportions in common populations in America. Hum Mutat 2009, 30:69-78.

7. Mao X, Bigham AW Mei R, Gutierrez G, Weiss KM, Brutsaert TD, LeonVelarde F, Moore LG, Vargas E, McKeigue PM, Shriver MD, Parra EJ: A genomewide admixture mapping panel for Hispanic/Latino populations. Am J Hum Genet 2007, 80:1171-1178.

8. Raaum RL, Wang AB, Al-Meeri AM, Mulligan CJ: Efficient population assignment and outlier detection in human populations using biallelic markers chosen by principal component-based rankings. BioTechniques 2010, 48:449-454.

9. Halder I, Shriver M, Thomas M, Fernandez JR, Frudakis T: A panel of ancestry informative markers for estimating individual biogeographical ancestry and admixture from four continents: utility and applications. Hum Mutat 2008, 29:648-658.

10. Phillips C, Salas A, Sánchez JJ, Fondevila M, Gómez-Tato A, Alvarez-Dios J, Calaza M, de Cal MC, Ballard D, Lareu MV, Carracedo A, SNPforlD Consortium: Inferring ancestral origin using a single multiplex assay of ancestry-informative marker SNPs. Forensic Sci Int Genet 2007, 1:273-280

11. Lao $O$, van Duijn K, Kersbergen $P$, de Knijff $P$, Kayser M: Proportioning whole-genome single-nucleotide-polymorphism diversity for the identification of geographic population structure and genetic ancestry. Am J Hum Genet 2006, 7:680-690.

12. Jorde LB, Wooding SP: Genetic variation, classification and "race". Nat Genet 2004, 36(Suppl):S28-S33.

13. Enoch MA, Shen PH, Xu K, Hodgkinson C, Goldman D: Using ancestryinformative markers to define populations and detect population stratification. J Psychopharmacol 2006, 20:199-126.

14. Shriver MD, Mei R, Parra EJ, Sonpar V, Halder I, Tishkoff SA, Schurr TG, Zhadanov SI, Osipova LP, Brutsaert TD, Friedlaender J, Jorde LB, Watkins WS, Bamshad MJ, Gutierrez G, Loi H, Matsuzaki H, Kittles RA, Argyropoulos G, Fernandez JR, Akey JM, Jones KW: Large-scale SNP analysis reveals clustered and continuous patterns of human genetic variation. Hum Genomics 2005, 2:81-89.

15. Shriver MD, Parra EJ, Dios $S$, Bonilla $C$, Norton $H$, Jovel $C$, Pfaff $C$, Jones $C$, Massac A, Cameron N, Baron A, Jackson T, Argyropoulos G, Jin L, Hoggart CJ, McKeigue P, Kittles RA: Skin pigmentation, biogeographical ancestry and admixture mapping. Hum Genet 2003, 112:387-399.

16. Paschou P, E Ziv E, Burchard EG, Choudhry S, Rodriguez-Cintron W, Mahoney MW, Drineas P: PCA-correlated SNPs for structure identification in worldwide human populations. PLOS Genet 2007, 3:e160.

17. Bauchet M, McEvoy B, Pearson LN, Quillen EE, Sarkisian T, Hovhannesyan K, Deka R, Bradley DG, Shriver MD: Measuring European population stratification with microarray genotype data. Am J Hum Genet 2007, 80:948-954.

18. Novembre J, Johnson T, Bryc K, Kutalik Z, Boyko AR, Auton A, Indap A King KS, Bergmann S, Nelson MR, Stephens M, Bustamante CD: Genes mirror geography within Europe. Nature 2008, 456:98-101, (Erratum in Nature 2008, 456:274.)

19. Moskvina V, Smith M, Ivanov D, Blackwood D, Stclair D, Hultman C, Toncheva D, Gill M, Corvin A, O'Dushlaine C, Morris DW, Wray NR, Sullivan P, Pato C, Pato MT, Sklar P, Purcell S, Holmans P, O'Donovan MC, 
Owen MJ, Kirov G: Genetic differences between five European populations. Hum Hered 2010, 70:141-149.

20. Fejerman L, Haiman CA, Reich D, Tandon A, Deo RC, John EM, Ingles SA, Ambrosone $C B$, Bovbjerg DH, Jandorf $L H$, Davis W, Ciupak $G$,

Whittemore AS, Press MF, Ursin G, Bernstein L, Huntsman S, Henderson BE, Ziv E, Freedman ML: An admixture scan in 1,484 African American women with breast cancer. Cancer Epidemiol Biomarkers Prev 2009, 8:3110-3117.

21. Bock CH, Schwartz AG, Ruterbusch JJ, Levin AM, Neslund-Dudas C, Land SJ, Wenzlaff AS, Reich D, McKeigue P, Chen W, Heath El, Powell IJ, Kittles RA, Rybicki BA: Results from a prostate cancer admixture mapping study in African-American men. Hum Genet 2009, 126:637-642.

22. Nelson MR, Bryc K, King KS, Indap A, Boyko AR, Novembre J, Briley LP, Maruyama Y, Waterworth DW, Waeber G, Vollenweider P, Oksenberg JR, Hauser SL, Stirnadel HA, Kooner JS, Chambers JC, Jones B, Mooser V, Bustamante CD, Roses AD, Burns DK, Ehm MG, Lai EH, The Population Reference Sample, POPRES: A resource for population, disease, and pharmacological genetics research. Am J Hum Genet 2008, 83:347-358.

23. Cann HM, deToma C, Cazes L, Legrand MF, Morel V, Piouffre L, Bodmer J, Bodmer WF, Bonne-Tamir B, Cambon-Thomsen A, Chen Z, Chu J,

Carcassi C, Contu L, Du F, Excoffier L, Ferrara GB, Friedlaender JS, Groot H, Gurwitz D, Jenkins T, Herrera RJ, Huang X, Kidd J, Kidd KK, Langaney A, Lin AA, Mehdi SQ, Parham P, Piazza A, Pistillo MP, Qian Y, Shu Q, Xu J, Zhu S, Weber JL, Greely HT, Feldman MW, Thomas G, Dausset J, CavalliSforza LL: A human genome diversity cell line panel. Science 2002, 296:261-262.

24. Kim JJ, Verdu P, Pakstis AJ, Speed WC, Kidd JR, Kidd KK: Use of autosomal loci for clustering individuals and populations of East Asian origin. Hum Genet 2005, 117:511-519.

25. Pakstis AJ, Speed WC, Kidd JR, Kidd KK: Candidate SNPs for a universal individual identification panel. Hum Genet 2007, 121:305-317.

26. Pakstis AJ, Speed WC, Fang R, Hyland FCL, Furtado MR, Kidd JR, Kidd KK: SNPs for a universal individual identification panel. Human Genetics 2010, 127:315-324.

27. Nassir R, Kosoy R, Tian C, White PA, Butler LM, Silva G, Kittles R, AlarconRiquelme ME, Gregersen PK, Belmont JW, De La Vega FM, Seldin MF: An ancestry informative marker set for determining continental origin: validation and extension using human genome diversity panels. BMC Genet 2009, 10:39.

28. HapMaplll. [http://hapmap.ncbi.nlm.nih.gov/index.html.yo].

29. Li JZ, Absher DM, Tang H, Southwick AM, Casto AM, Ramachandran S, Cann HM, Barsh GS, Feldman M, Cavalli-Sforza LL, Myers RM: Worldwide human relationships inferred from genome-wide patterns of variation. Science 2008, 319:1100-1104.

30. ALFRED, the Allele Frequency Database. [http://alfred.med.yale.edu/alfred/ AboutALFRED.asp].

31. Stanford HGDP SNP Genotyping Data. [http://hagsc.org/hgdp/files.html].

32. Wright S: Evolution and the Genetics of Populations, vol 2: The Theory of Gene Frequencies Chicago and London: University of Chicago Press; 1969.

33. Pritchard JK, Stephens M, Donnelly P: Inference of population structure using multilocus genotype data. Genetics 2000, 155:945-959.

34. Falush D, Stephens M, Pritchard JK: Inference of population structure: extensions to linked loci and correlates allele frequencies. Genetics 2003, 164:1567-1587.

35. Falush D, Stephens M, Pritchard JK: Inference of population structure using multilocus genotype data: dominant markers and null alleles. $\mathrm{Mol}$ Ecol Notes 2007, 7:574-578.

36. Jakobsson M, Rosenberg NA: CLUMPP: a cluster matching and permutation program for dealing with label switching and multimodality in analysis of population structure. Bioinformatics 2007, 23:1801-1806

37. Rosenberg NA: DISTRUCT: a program for the graphical display of population structure. Mol Ecol Notes 2004, 4:137-138.

38. Rosenberg NA: Algorithms for selecting informative marker panels for population assignment. J Comput Biol 2005, 12:1183-1201.

39. Rosenberg NA, Li LM, Ward R, Pritchard JK: Informativeness of genetic markers for inference of ancestry. Am J Hum Genet 2003, 73:1402-1422.

40. Royal CD, Novembre J, Fullerton SM, Goldstein DB, Long JC, Bamshad MJ, Clark AG: Inferring genetic ancestry: opportunities, challenges, and implications. Am J Hum Genet 2010, 86:661-673.
41. Kalinowski ST: The computer program STRUCTURE does not reliably identify the main genetic clusters within species: simulations and implications for human population structure. Heredity 2010.

doi:10.1186/2041-2223-2-1

Cite this article as: Kidd et al: Analyses of a set of 128 ancestry informative single-nucleotide polymorphisms in a global set of 119 population samples. Investigative Genetics 2011 2:1.

\section{Submit your next manuscript to BioMed Central and take full advantage of:}

- Convenient online submission

- Thorough peer review

- No space constraints or color figure charges

- Immediate publication on acceptance

- Inclusion in PubMed, CAS, Scopus and Google Scholar

- Research which is freely available for redistribution

Submit your manuscript at www.biomedcentral.com/submit
Biomed Central 